\title{
Identification of sleep disruption and sleep disordered breathing from the systolic blood pressure profile
}

\author{
R J O Davies, K Vardi-Visy, M Clarke, J R Stradling
}

\begin{abstract}
Background-Respiratory sleep studies are frequently performed to identify sleep disruption resulting from upper airway obstruction. Traditional polysomnographic studies may not detect brief recurrent sleep disruption and thus fail to recognise a significant problem when apnoea, hypopnoea, or arterial desaturation are not present. Arousal from sleep causes a transient blood pressure rise, and each inspiration causes a transient blood pressure fall. This study assesses whether these blood pressure changes are a useful indirect marker of disturbed sleep, obstructed sleep apnoea, and snoring related sleep disturbance.

Methods-Computer algorithms were developed to identify blood pressure falls caused by inspiration and rises related to arousal from 286 sleeping blood pressure samples of a consistent respiratory state drawn from 51 polysomnographic studies. From these samples, normal ranges for the number of arousal related systolic rises and the average size of the inspiratory falls were established. These were then applied prospectively to all night unedited blood pressure recordings from a further 20 subjects.
\end{abstract}

Results-The size of the inspiratory falls in blood pressure progressively increased from normal sleep, through snoring, to frank obstructive sleep apnoea. The 95th centile of normal was $12.5 \mathrm{~mm} \mathrm{Hg}$. The number of arousal related blood pressure rises also increased during obstructive sleep apnoea and periods of snoring with associated arousals, compared with normal undisturbed sleep, and all these periods of disturbed sleep included more than 30 such rises per hour. When these blood pressure features were examined in the 20 subjects studied prospectively, the six with a sleep related breathing disorder could all have been identified from their systolic blood pressure profile alone.

Conclusions-The systolic blood pressure profile may be helpful in identifying patients with obstructive sleep apnoea, snoring with arousals, or other sleep disruption syndromes.

(Thorax 1993;48:1242-1247)
Obstructive and central sleep apnoea, ${ }^{1}$ snor- $\vec{\circ}$ ing with arousal from sleep, ${ }^{23}$ and nocturnal periodic movements of the legs $^{4}$ all cause $\vec{\omega}$ symptomatic sleep disruption which is $\stackrel{\mathscr{F}}{\vec{F}}$ reversible with appropriate treatment. The $\vec{x}$ traditional way of assessing these disorders is $\vec{\infty}$ by polysomnography ${ }^{5}$ which includes sleep $\vec{\sim}$ staging from the electroencephalogram $\vec{N}$ (EEG), electro-occulogram (EOG), and electromyogram (EMG), and respiratory moni- $\circ$ toring, usually from oronasal airflow, arterial oxygen saturation, and an index of respiratory 0 effort. Such complex studies are time con- $\mathbb{D}_{\infty}^{\circ}$ suming and tend to disturb normal sleep $\stackrel{\oplus}{3}$ (changing the state they are attempting to $\underset{\mathbb{\Phi}}{\mathbb{S}}$ document) ${ }^{6}$

Simpler systems, usually based on an arter- $\bar{\varnothing}$ ial pulse oximeter, are available and are ${ }^{\omega}$ designed to identify frank obstructive sleep apnoea. It has recently been shown, however, that severe symptomatic sleep disruption which reverses with nasal continuous positive $\stackrel{0}{\circ}$ airway pressure can occur without apnoea, $\stackrel{\circ}{\circ}$ hypopnoea, or oximetry abnormality in $\overrightarrow{\overrightarrow{0}}$ response to the increased inspiratory effort of 3 snoring alone. ${ }^{23}$ These patients (as well as those with non-respiratory sleep disruption) would appear normal if only respiratory indices were considered.

The systolic blood pressure falls with inspiration, ${ }^{7}$ and rises with arousal from 3 . sleep. ${ }^{8}$ The size of these inspiratory falls is a semiquantitative indicator of the degree of respiratory effort, ${ }^{9}$ and the rises coincident 0 with arousal are a consistent feature of sleep disruption syndromes including obstructive sleep apnoea, ${ }^{10}$ central sleep apnoea, ${ }^{11}$ and $\underset{N}{\sim}$ nocturnal periodic movements of the legs. ${ }^{12}$ o Non-invasive blood pressure measurement $\tilde{O}$ could therefore be used to identify abnormal $\mathbb{\omega}$ respiration or sleep disturbance without the need for traditional polysomnography.

This study aims to assess whether the systolic blood pressure profile could have a role in the assessment of patients with possible obstructive sleep apnoea.

Methods

The study was performed in two parts. The 8 systolic blood pressure characteristics which? best defined inspiratory blood pressure falls

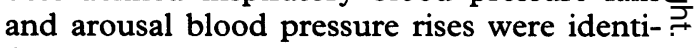
fied from carefully selected samples of normal sleeping respiration, snoring (with and with- 
out arousals), and obstructive sleep apnoea. These characteristics were then used in a computer algorithm and applied prospectively to sleeping blood pressure recordings from patients having traditional polysomnography for possible obstructive sleep apnoea. The study was approved by the Central Oxford Research Ethics Committee.

DEVELOPMENT OF THE COMPUTER PROGRAMS Representative samples of sleeping blood pressure were drawn from 17 normal volunteers who neither snored nor had obstructive sleep apnoea, 13 confirmed snorers, and 21 subjects with obstructive sleep apnoea.

These recordings were made during a polysomnogram (EEG C3/A2, EOG $\times 2$, chin EMG, rib cage and abdomen movement (inductance plethysmography), oronasal air- flow (thermistors)-MPA 2/Medilog 9200, Oxford Medical Instruments, Abingdon, UK-pulse oximetry, and audio/video recording). Sleep was staged according to standard criteria. ${ }^{13}$ Arousal from sleep was defined as speeding of the EEG for at least three seconds with an associated rise in EMG. Snoring was identified from the audio recording. Arterial blood pressure was recorded using a finger infrared light absorbency method (Finapres; Ohmeda, Englewood, Colorado, USA) logged to an IBM compatible PC, and synchronised with the sleep and respiratory data.

Multiple periods of constant respiratory and sleep state were selected from these studies. Each selected period satisfied the following criteria:

1. Duration of five to 10 minutes.

2. A consistent non-rapid eye movement
Figure 1 Arterial blood pressure profile during two cycles of obstructive sleep apnoea before and after smoothing of the systolic blood pressure with a moving five beat mean. Both the falls with inspiration and the rises with arousal are visible in the top trace. The lower trace is smoothed to removed the inspiratory blood pressure falls. Small residual inspiratory falls (A) persist despite the smoothing (see text).

Three of the arousal related blood pressure rises (B) and three of the inspiratory falls before smoothing $(C)$ are indicated.
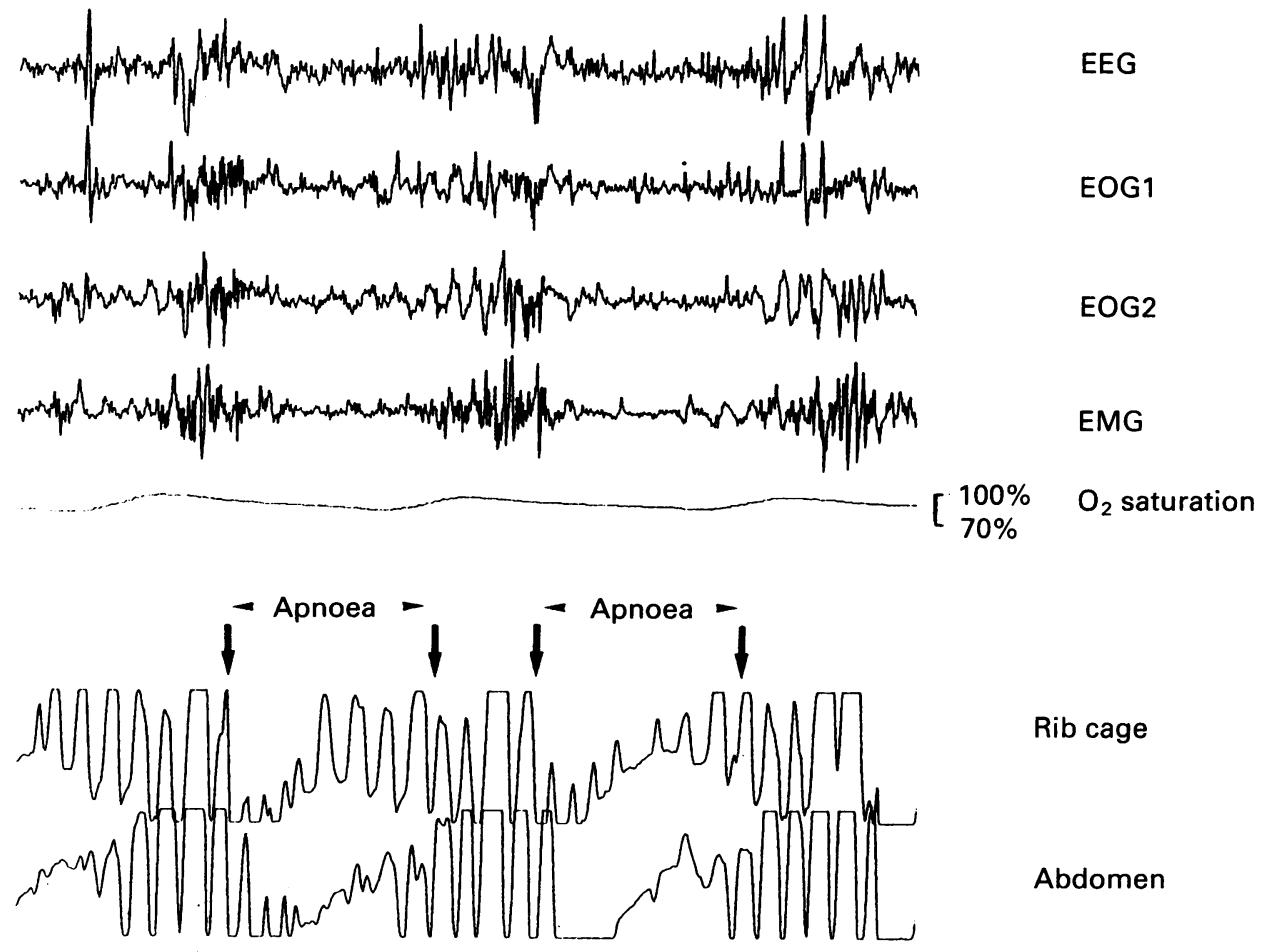

Rib cage

Abdomen

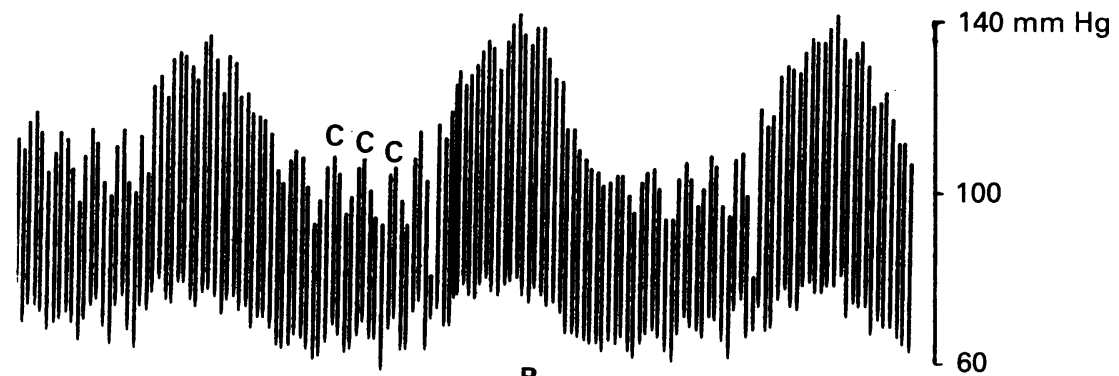

Unsmoothed blood pressure

Smoothed blood pressure 
sleep stage. For periods of normal sleep or snoring without arousals one level of sleep state change was allowed on 30 second epoch sleep staging ${ }^{13}$ - that is, $1 / 2,2 / 3,3 / 4$. For periods where the sleep architecture was fragmented by respiratory disturbance, the period was acceptable provided the period included no REM sleep.

3. A consistent respiratory state. The period consisted of continuous normal sleep, snoring (one or no EEG arousals), snoring with arousals (more than five EEG arousals), or obstructive sleep apnoea. Snoring with arousals and oxygen desaturation but no frank apnoea was classified as snoring with arousals.

4. The hand bearing the Finapres remained at a consistent hydrostatic level relative to the heart (assessed from the audio/video recording).

5. The blood pressure trace was not affected by movement artifact or interruption of the trace.

All the periods which satisfied these criteria were used in the analysis, and the respiratory state represented by each sample was decided before its blood pressure profile was examined.

The raw data files from five of these samples were visually reviewed and the time course which described the blood pressure falls in time with inspiration was identified. This provisional definition was then applied to five further short samples and its results compared with manual identification of the respiratory falls from the same traces. The causes of any discrepancies were identified and the algorithms refined as a result. This "test and adapt" approach was repeated until the optimal definition was derived. The blood pressure profile was then smoothed with a moving five beat mean to facilitate identification of the arousal related rises (fig 1) and the characteristics which best described these rises similarly defined. These digital algo-
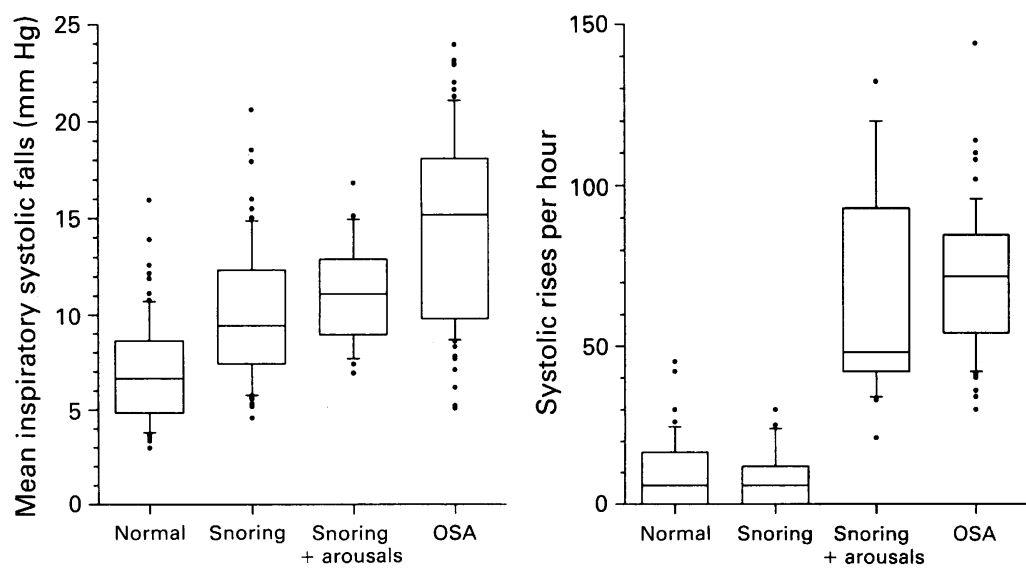

Respiratory state

Figure 2 Box and whisker plots of the mean inspiratory systolic falls and arousal related rises during selected periods of normal sleep, snoring (with and without arousals), and obstructive sleep apnoea (OSA). Since the data are not normally distributed, the plots show the median, the interquartile range, and the 10th and 90th centiles. Individual data points which lie outside the 10th and 90th centiles are individually shown. rithms were used to extract the size of the inspiratory falls, and to count and quantify the arousal related rises from all the short samples of sleeping blood pressure.

\section{PROSPECTIVE TESTING OF THE COMPUTER} PROGRAMS

The programs capable of identifying blood $\stackrel{x}{x}$ pressure falls due to inspiration and rises at $\vec{F}$ arousal were combined to produce one pro- $\stackrel{5}{+}$ gram which processed a whole night's blood pressure recording. This program was then $\frac{\sigma}{\bar{c}}$ applied to records from 20 new patients being $\frac{\widehat{\sigma}}{\overparen{\sigma}}$ assessed for possible obstructive sleep apnoea. The blood pressure recordings from $\mathrm{c}$ these subjects were not edited to remove $\overrightarrow{0}$ movement artifacts and the analysis was per- $\vec{\overrightarrow{ }}$ formed on the whole of the record. Each ${ }_{\sigma}^{\omega}$ patient completed a questionnaire document- $\overrightarrow{\vec{v}}$ ing the symptoms of obstructive sleep apnoea $x$ and daytime sleepiness. ${ }^{14}$ The patients then $\$$ underwent polysomnographic measurements $\vec{N}$ similar to those used during the retrospective $\vec{N}$ data collection. The severity of obstructive $\hat{N}$ sleep apnoea was quantified from these응 studies as the number of $>4 \%$ dips in arterial saturation per hour of sleep.

These 20 subjects were divided into three $\varnothing_{\varnothing}^{\mathbb{R}}$ clinical groups on the basis of their clinical ${ }^{\circ}$ features (from the questionnaires), and their $\mathbb{\Phi}$ polysomnographic results. This decision was $\vec{\bullet}$ made blind of the blood pressure data. The categories were: (1) normal (quiet undisturbed sleep and no abnormal oxygen desaturation, apnoea, or hypopnoea); (2) snorer (history of snoring, snoring during sleep without recurrent arousals from sleep, and no abnormal oxygen desaturation, apnoea, or $\stackrel{\varrho}{\Rightarrow}$ hypopnoea); (3) significant sleep related윽 breathing disorder (history of sleepiness and snoring, together with periods of heavy snoring with recurrent EEG arousals or abnormal oxygen saturation dipping, apnoea, or hypo-음 pnoea, or both).

After the patients had been allocated to one of the groups, the blood pressure profile 3 . was analysed and the ability of the blood pressure analysis to predict the severity of the obstructive sleep apnoea, and the clinicalo diagnostic and treatment decisions were examined.

\section{Results}

RETROSPECTIVE DEVELOPMENT OF THE COMPUTER ALGORITHM

A total of 286 sleep periods were identified from the 51 polysomnograms, 72 of which were normal sleep, 98 snoring, 25 snoring with EEG arousals, and 91 obstructive sleepo apnoea.

The optimal description of an inspiratory related fall in systolic blood pressure was found to be any fall from peak to trough lasting more than 1.5 seconds but less than 7.50 seconds. The optimal description of anarousal related rise was found to be a rise of more than $18 \mathrm{~mm} \mathrm{Hg}$ which ended in a fall of $4 \mathrm{~mm} \mathrm{Hg}$, and which lasted more than $4 \mathrm{sec}-$ onds and less than 45 seconds. The terminal 


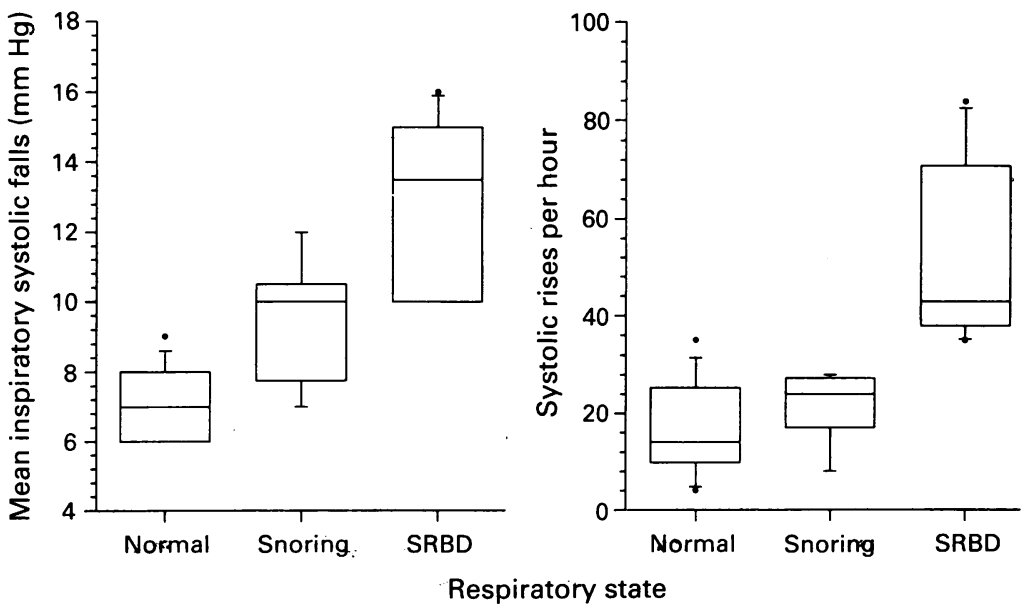

Figure 3 Box and whisker plots of the mean inspiratory systolic falls and arousal related rises during all night unedited blood pressure traces gathered during the prospective study. Since the data are not normally distributed, the plots show the median, the interquartile range, and the 10th and 90th centiles. Individual data points which lie outside the 10th and 90th centiles are individually shown. SRBD—sleep related breathing disorder.

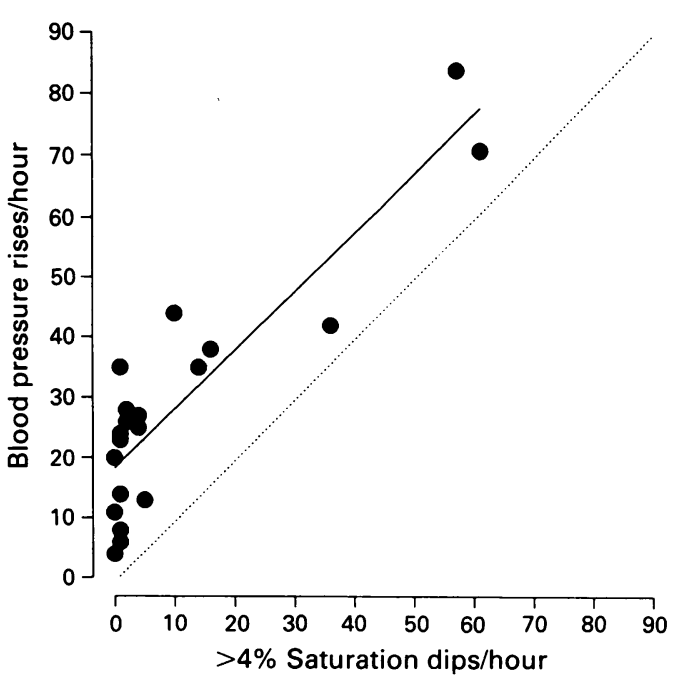

Figure 4 Number of $>4 \%$ oxygen saturation dips and the number of arousal related blood pressure rises per hour in the prospective study group. The solid line shows the line of regression and the dotted line shows the line of identity.

fall of $4 \mathrm{~mm} \mathrm{Hg}$ was introduced to allow for small inspiratory blood pressure falls which persisted despite the smoothing process (fig 1). The $18 \mathrm{~mm} \mathrm{Hg}$ threshold was selected to optimally separate blood pressure rises associated with arousal from the spontaneous changes which occur during normal sleep, particularly the blood pressure rises which accompany $\mathrm{K}$ complexes. ${ }^{15}$

By these criteria the falls in systolic blood pressure related to inspiration progressively increased from normal sleep, through snoring and snoring with arousals, to frank obstructive sleep apnoea (fig 2). The number of arousal associated blood pressure rises also increased across these diagnostic categories (fig 2). Of the 117 periods of sleep characterised by either snoring with arousals or frank obstructive sleep apnoea, 116 included more than 30 blood pressure rises per hour. Only two periods of undisturbed sleep (normal sleep and snoring without arousals) included blood pressure rises above this threshold (fig 2). Both these samples were of light (stage 1/2) sleep, and recurrent EEG arousals were present but were not caused by a respiratory abnormality.

From these analyses two arbitrary definitions of normality were selected for use in the prospective analysis; the 95th centile for the inspiratory falls in blood pressure for the normal samples was set at $12.5 \mathrm{~mm} \mathrm{Hg}$ (fig 2), and the threshold for blood pressure rises was fixed at 30 per hour since all but one of the periods of clinically abnormal sleep had values above this limit (fig 2 ).

\section{PROSPECTIVE TESTING OF THE COMPUTER PROGRAMS}

The characteristics of the 20 subjects studied prospectively are shown in the table. Nine were considered normal, five snored without significant sleep disruption, and six had a significant sleep related breathing disorder. All the subjects with significant sleep disordered breathing were offered treatment. In five cases this was a trial of nasal continuous positive airway pressure and in one case tonsillectomy. All six subjects considered to have a significant sleep and breathing disorder had $>4 \%$ arterial oxygen saturation dip rates above 10 per hour.

The mean size of the inspiration related falls in systolic blood pressure increased across each of the three diagnostic categories (fig 3; p $<0.0001$, analysis of variance and Duncan's post hoc multiple range test). The number of blood pressure rises suggesting arousal from sleep was greater in the group with significant sleep disordered breathing than it was in the normal subjects or snorers without arousals (fig 3; p $<0.004$, analysis of variance and Duncan's post hoc multiple range test).

All six subjects with a sleep related breathing disorder had more than 30 blood pressure rises per hour of the study. One other subject also exceeded this limit. As a method of identifying subjects with obstructive sleep apnoea or snoring induced arousals, the number of blood pressure rises per hour of sleep was

Median (range) characteristics of the 20 subjects included in the prospective study

\begin{tabular}{llll}
\hline & $\begin{array}{l}\text { Normal subjects } \\
(n=9)\end{array}$ & $\begin{array}{l}\text { Simple snorers } \\
(n=5)\end{array}$ & $\begin{array}{l}\text { Sleep related } \\
\text { breathing disorder } \\
(n=6)\end{array}$ \\
\hline Age (years) & $51(30-66)$ & $40(37-74)$ & $50(43-62)$ \\
M:F & $8: 1$ & $4: 1$ & $5: 1$ \\
Body mass index $\left(\mathrm{kg} / \mathrm{m}^{2}\right)$ & $27 \cdot 9(22 \cdot 9-33 \cdot 2)$ & $28 \cdot 1(24 \cdot 6-31 \cdot 9)$ & $35 \cdot 5(26 \cdot 5-43 \cdot 1)$ \\
Neck circumference $(\mathrm{cm})$ & $41 \cdot 9(35 \cdot 7-44 \cdot 5)$ & $40 \cdot 6(38 \cdot 1-44 \cdot 4)$ & $46 \cdot 3(39 \cdot 3-51 \cdot 0)$ \\
$>4 \%$ oxygen saturation dip rate/hour & $1(0-5)$ & $2(1-6)$ & $59(10 \cdot 2-71)$ \\
\hline
\end{tabular}


thus $100 \%$ sensitive and $87 \%$ specific. The positive predictive value of an abnormal result was $75 \%$, and the negative predictive value of a normal result $100 \%$.

Four of the seven subjects with more than 30 blood pressure rises per hour had a mean inspiratory blood pressure fall of more than $12.5 \mathrm{~mm} \mathrm{Hg}$, and all these subjects had a sleep related breathing disorder. Of the three others, one was normal and two had obstructive sleep apnoea with small associated inspiratory efforts on the video and respiratory movement recordings.

The relationship between the $>4 \%$ arterial oxygen saturation dip rate and the number of arousal related blood pressure rises is shown in fig 4. These indices correlated moderately well $(r=0.88,95 \%$ CI 0.72 to $0.95, \mathrm{p}<$ 0.0001 ), but there were consistently more blood pressure "events" than desaturation "events" (range of differences 4-34 per hour).

\section{Discussion}

This study has shown that simple computer algorithms can identify the falls in blood pressure which occur with sleeping respiration and the rises which occur when sleep is fragmented by a respiratory abnormality. The number of arousal related rises is a useful marker for identifying patients with pathologically disturbed sleep, and the inspiratory related falls are helpful in isolating an obstructive respiratory cause for this in about half the abnormal cases.

During this study beat-to-beat blood pressure was recorded with the Finapres device. This method depends on a pneumatic servocontrolled cuff which is inflated to keep the infrared absorption of the finger constant by correcting a photoplethysmogram. Since changes in digital infrared absorption are caused by variations in digital blood volume, ${ }^{16}$ the changes in cuff pressure required to keep the photoplethysmogram constant reflect changes in intravascular pressure. ${ }^{16}{ }^{17}$ This is an accurate method for monitoring blood pressure changes, and successfully follows the alterations which occur during the Valsalva manoeuvre, the Müller manoeuvre, atrial fibrillation, ${ }^{18}$ as well as obstructive sleep apnoea. ${ }^{19}$ The non-invasive nature of this method has obvious advantages in sleep study work and, being painless, may disrupt sleep less than invasive cannulation. Since digital arterial pressure differs from brachial arterial pressure because of the hydrostatic effects of hand position, ${ }^{1819}$ and the normal change in blood pressure along the vascular tree, ${ }^{20}$ the Finapres is best used as an index of blood pressure change, rather than of absolute pressure. ${ }^{18}$ For this reason all the blood pressure algorithms used in this study depend on monitoring blood pressure changes and no absolute pressures are used.

Patients with heavy snoring and sleep arousals can present with symptoms indistinguishable from classical obstructive sleep apnoea which respond to continuous positive airway pressure. ${ }^{23}$ For this reason the definition of a sleep related breathing disorder used in the prospective study allowed for the inclusion of subjects who snored, were sleepy, and demonstrated recurrent arousals during sleep, but whose other respiratory indices were nor-국 mal. In practice none of the 20 prospective subjects was included in this category on the basis of snoring with arousals alone, and all $\overrightarrow{\vec{F}}$ those classified as abnormal had an arterialo oxygen saturation dip rate of more than 10 음 per hour.

In this report a non-EEG marker of arousal $\frac{\widehat{\Phi}}{\Omega}$ has been studied. Such markers are simple to quantify and should be abnormal whatever ${ }_{\vec{A}}$ the cause of the sleep disturbance. They are? also a logical end point since the dominant $\vec{\omega}$ symptoms of the sleep apnoea syndromes are symptoms of sleep deprivation and not $\vec{x}$ hypoxaemia. ${ }^{21}$ Possible markers of arousal $\stackrel{+}{\infty}$ include movement, ${ }^{22}$ and autonomic changes $\vec{N}$ such as cardioacceleration ${ }^{23}$ or the blood pres- sure rise examined here. Movement is $a_{+}^{\vec{N}}$ marker of substantial arousal and correlates ${ }_{0}^{\mathrm{N}}$ with the presence of arousals. ${ }^{22}$ Unfortunately sustained wakefulness is associated with a lot of movement, and therefore a poor night'sष sleep with long periods of wakefulness pro- $\overparen{\Phi}$ duces very abnormal results, even in an뭉 otherwise normal subject. The autonomic $\stackrel{\mathbb{D}}{-}$ indices do not share this problem. Repetitive $\vec{\theta}$ arousal stimuli which cause significant autonomic changes during sleep do not produce similar changes during sustained wakefulness. ${ }^{24}$ Either blood pressure or heart rate might be a useable autonomic marker; how-ڤ ever, blood pressure responses do seem to be $\frac{\alpha}{\mathbb{D}}$

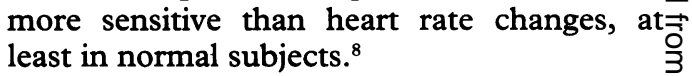

Some disadvantages of blood pressure measurement include the considerable num-0. ber of blood pressure rises during normal sleep, and the inconvenience of the large음 Finapres machine needed for its measure- $-\dot{x}$ ment. The large number of blood pressure $\frac{0}{3}$ rises during "normal" sleep is probably. explained by two mechanisms. Firstly, even normal sleep can be punctuated by about 20 transient arousals per hour. ${ }^{25}$ This was the explanation for the two "normal" periods with more than 30 blood pressure rises per hour of sleep in the retrospective study (figo 2). Secondly, some periods of undisturbedn sleep are characterised by repeated transientw్ blood pressure rises. This is particularly true of stage 2 sleep when $\mathrm{K}$ complexes are associ $-\overline{0}$ ated with downregulation of the arteriak baroreflex, ${ }^{26}$ a burst of sympathetic activity, ${ }^{2 \pi n} ?$ and a blood pressure rise ${ }^{26}$ which can some- $T$ times be large enough to exceed the $18 \mathrm{~mm}$ $\mathrm{Hg}$ threshold included in this study.

The results of the prospective testing of the $\frac{\vec{D}}{2}$ blood pressure software are encouraging. Counting the number of blood pressure rises from all night blood pressure traces identifiedo all the subjects with a sleep related breathing disorder. For this prospective study noattempt was made to select "clean" periods of the recording, as was done during the retrospective data collection. The computer soft- 
ware was applied to the whole record, including any periods of REM sleep, wakefulness and movement. Despite this, the results remained informative, probably because the small amount of "noise" introduced by occasional body movements was insufficient to obscure the result of the overall analysis. One normal subject showed more than 30 blood pressure rises per hour of the sleep study. This subject did indeed have unusually large blood pressure rises during normal light sleep and REM. This suggests that a small number of normal subjects are indistinguishable from those with pathological sleep disturbance on the basis of their blood pressure alone.

In four of the eight cases with an increased number of blood pressure rises the mean size of the inspiratory related blood pressure falls was above the 95th centile of normal. In all these subjects the sleep disruption was caused by upper airway narrowing or collapse. In these subjects a presumptive diagnosis of a sleep and breathing disorder could have been made from the blood pressure trace alone and a trial of nasal continuous positive airway pressure tried without further investigation. Therefore 17 of the 20 subjects $(85 \%)$ in this prospective group could have been classified as either normal or very likely to have a sleep related breathing disorder from their blood pressure recording alone. If this success rate is reproduced in larger samples, the blood pressure profile could reduce the need for more detailed sleep studies by four fifths in the population attending sleep clinics. This technique will need comparison (and perhaps combination) with other simple sleep study approaches such as video recordings with oximetry before its role in the sleep laboratory is finally established.

In conclusion, this study has shown that sleep related breathing disorders are associated with characteristic arterial blood pressure disturbances which are potentially useful as a screening tool for these disorders. Since the blood pressure profile includes information about sleep fragmentation as well as respiratory effort, this approach has theoretical advantages over systems which use purely respiratory variables. In particular, the information about sleep fragmentation should allow the identification of snoring induced arousals, and non-respiratory sleep disruption syndromes such as periodic movements of the legs.

This research was funded by the Wellcome Trust through a Graduate Research Fellowship awarded to Dr Davies, and a Senior Research Fellowship awarded to Dr Stradling. We would like to thank Ohmeda for their support with finger cuffs for the Finapres.

1 Guilleminault C, Tilkian A, Dement WC. The sleep apnea syndromes. Ann Rev Med 1976;27:465-84.

2 Guilleminault C, Stoohs R, Duncan S. Snoring (I). Daytime sleepiness in regular heavy snorers. Chest 1991; 99:40-8.
3 Hillerdal G, Hetta J, Lindholm CE, Hultcrantz E, Boman G. Symptoms in heavy snorers with and withou obstructive sleep apnea. Acta Otolaryngol Stockh 1991, 111:574-81.

4 Montplaisir J, Godbout R. Restless legs syndrome and periodic movements during sleep. In: Kryger $M H$, Roth T, Dement WC. eds. Principles and practice of sleep medicine. Philadelphia: Saunders, 1989:402-9.

5 Martin RJ, Block AJ, Cohn MA, Conway WA, Hudgel DW, Powles ACP, et al. Indications and standards for cardiopulmonary sleep studies. Sleep 1985;8:371-9.

6 Mendels J, Hawkins DR. Sleep laboratory adaptation in normal subjects and depressed patients ("first night effect"). Electroencephalogr Clin Neurophysiol 1967;22 556-8.

7 Karam M, Wise RA, Natarajan TK, Permutt S, Wagner HN. Mechanism of decreased left ventricular stroke volume during inspiration in man. Circulation 1984; 69:866-73.

8 Davies RJO, Belt PJ, Robert SJ, Ali NJ, Stradling JR. Arterial blood pressure responses to graded transien arousal from sleep in normal humans. $\mathcal{F}$ Appl Physio 1993;74:1123-30

9 Lea S, Ali NJ, Goldman M, Loh L, Fleetham J, Stradling JR. Systolic blood pressure swings reflect inspiratory effort during simulated obstructive sleep apnoea. In Horne J, ed. Sleep 90. Bochum: Pontenagel Press, 1990:178-81.

10 Shepard JW. Gas exchange and hemodynamics during sleep. Med Clin North Am 1985;69:1243-64.

11 Davies RJO, Ali NJ, Fleetham J, Stradling JR. Observations on the mechanisms of blood pressure swings during central sleep apnoea and periodic movement of the legs. Am Rev Respir Dis 1991;143:383.

12 Ali NJ, Davies RJ, Fleetham JA, Stradling JR. Periodic movements of the legs during sleep associated with rises in systemic blood pressure. Sleep 1991;14:163-5.

13 Rechtschaffen A, Kales A. A manual of standardised terminology, techniques and scoring system for sleep stages of human subjects. Washington DC: National Institutes of Health, Publication No 204, 1968

14 Davies RJO, Ali NJ, Stradling JR. Neck circumference and other clinical features in the diagnosis of the obstructive sleep apnoea syndrome. Thorax 1992;47: 101-5.

15 Coccagna G, Mantovani M, Brignani F, Manzini A, Lugaresi E. Arterial pressure changes during spontaneous sleep in man. Electroencephalogr Clin Neurophysio 1971;31:277-81.

16 Penaz J. Photoelectric measurement of blood pressure volume and flow in the finger. Digest of the international conference on medicine and biological engineering. Dresden: International Conference on Biomedical Engineering, 1973:104-5.

17 Boehmer RD. Continuous, real-time, noninvasive monitor of blood pressure: Penaz methodology applied to the finger. $\mathcal{F}$ Clin Monit 1987;3:282-7.

18 Parati G, Casadei R, Groppelli A, Di Rienzo M, Mancia $G$. Comparison of finger and intra-arterial blood pressure monitoring at rest and during laboratory testing Hypertension 1989;13:647-55.

19 Penzel T, Ducke E, Peter JH, Podzus T, Schneider H, Stahler $\mathrm{J}$, et al. Non-invasive monitoring of blood pressure in a sleep laboratory. In: Ruddel H, Curio I, eds. Non-invasive continuous blood pressure measurement. Paris: Peter Lang, 1991:95-106.

20 Smith NT, Wesseling KH, de-Wit B. Evaluation of two prototype devices producing noninvasive, pulsatile, calibrated blood pressure measurement from a finger. $7 \mathrm{Clin}$ Monit 1985;1:17-29.

21 Roehrs T, Zorick F, Wittig R, Conway W, Roth $T$. Predictors of objective level of daytime sleepiness in patients with sleep-related breathing disorders. Chest 1989;95:1202-6.

22 Vos PJE, Stradling JR. Assessment of sleep times and movement arousals from video recordings. I Ambul Monit 1991;4:35-42.

23 Rauscher H, Popp W, Zwick H. Quantification of sleep disordered breathing by computerised analysis of oximetry, heart rate and snoring. Eur Respir f 1991;4:655-9.

24 Johnson LC, Lubin A. The orienting reflex during waking and sleeping. Electroencephalogr Clin Neurophysiol 1967 22:11-21.

25 Anholm JD, Powles AC, Downey R, Houston CS, Sutton JR, Bonnet MH, et al. Operation Everest II: arterial oxyJen saturation and sleep at extreme simulated altitude. gen saturation and sleep at extreme

26 Smyth HS, Sleight P, Pickering GW. Reflex regulation of arterial pressure during sleep in man. Circ Res 1969 14:109-21.

27 Hornyak $M$, Cejnar $M$, Matousek $M$, Wallin BG. Sympathetic muscle nerve activity during sleep in man. Brain 1991;114:1281-95. 